\title{
CHARACTERIZATION OF A CLASS OF EMBEDDINGS FOR FUNCTION SPACES WITH MUCKENHOUPT WEIGHTS
}

\author{
MARTIN MEYRIES AND MARK VERAAR
}

\begin{abstract}
For function spaces equipped with Muckenhoupt weights, the validity of continuous Sobolev embeddings in case $p_{0} \leq p_{1}$ is characterized. Extensions to Jawerth-Franke embeddings, vector-valued spaces and examples involving some prominent weights are also provided.
\end{abstract}

\section{INTRODUCTION}

This note is concerned with the characterization of continuous embeddings for function spaces equipped with Muckenhoupt weights $w$, and their vector-valued extensions. We consider Besselpotential spaces $H^{s, p}\left(\mathbb{R}^{d}, w\right)$, Sobolev-Slobodetskii spaces $W^{s, p}\left(\mathbb{R}^{d}, w\right)$, Triebel-Lizorkin spaces $F_{p, q}^{s}\left(\mathbb{R}^{d}, w\right)$ and Besov spaces $B_{p, q}^{s}\left(\mathbb{R}^{d}, w\right)$. Definitions, basic properties and references to systematic investigations of these spaces and Muckenhoupt's $A_{p}$-classes are given in Section 2

Sharp embeddings of Sobolev type are fundamental for these function spaces and their applications. In the unweighted case, characterizations of their validity in terms of the parameters are classical, see [29]. In the presence of Muckenhoupt weights, the following characterization for Besov spaces was obtained in [12, Proposition 2.1], via wavelet representations of Besov norms and the corresponding discrete characterizations from [15, Theorem 3.1].

Proposition 1.1 (Haroske \& Skrzypczak [12]). Let $w_{0}, w_{1} \in A_{\infty}, s_{0} \geq s_{1}, 0<p_{0}, p_{1} \leq \infty$ and $0<q_{0}, q_{1} \leq \infty$. Define $0<p_{*}, q_{*} \leq \infty$ by

$$
\frac{1}{p_{*}}=\left(\frac{1}{p_{1}}-\frac{1}{p_{0}}\right)_{+} \text {and } \frac{1}{q_{*}}=\left(\frac{1}{q_{1}}-\frac{1}{q_{0}}\right)_{+} .
$$

Then there is a continuous embedding

$$
B_{p_{0}, q_{0}}^{s_{0}}\left(\mathbb{R}^{d}, w_{0}\right) \hookrightarrow B_{p_{1}, q_{1}}^{s_{1}}\left(\mathbb{R}^{d}, w_{1}\right)
$$

if and only if

$$
\left\|\left(2^{-\nu\left(s_{0}-s_{1}\right)}\left\|\left[w_{0}\left(Q_{\nu, m}\right)^{-1 / p_{0}} w_{1}\left(Q_{\nu, m}\right)^{1 / p_{1}}\right]_{m \in \mathbb{Z}^{d}}\right\|_{\ell^{p_{*}}}\right)_{\nu \in \mathbb{N}_{0}}\right\|_{\ell^{q_{*}}}<\infty .
$$

Here $r_{+}=\max \{r, 0\}, \ell^{p}$ are the usual sequence spaces, $Q_{\nu, m} \subset \mathbb{R}^{d}$ denotes for $\nu \in \mathbb{N}_{0}$ and $m \in \mathbb{Z}^{d}$ the $d$-dimensional cube with sides parallel to the coordinate axes, centered at $2^{-\nu} m$ and with side length $2^{-\nu}$, and further $w(Q)=\int_{Q} w(x) d x$ for a weight $w$ and a cube $Q$. Observe that in the above result, for $p_{0} \leq p_{1}$ and $q_{0} \leq q_{1}$ one has $p_{*}=q_{*}=\infty$. It is interesting to note that the condition (1.1) improves if one restricts to radially symmetric distributions, even though the weights can be arbitrary, see [6].

The purpose of this paper is to provide similar characterizations in case $p_{0} \leq p_{1}$ for other types of the above mentioned weighted spaces, for embeddings of mixed type, and for the vector-valued case. Our main result concerns Triebel-Lizorkin spaces. Its proof, given in Section 3.2, is based on Proposition 1.1 and a weighted Gagliardo-Nirenberg inequality. It is also the basis for the other types of embeddings in Theorem 1.3 below.

2000 Mathematics Subject Classification. 46E35, 46E40.

Key words and phrases. Sobolev embeddings, Jawerth-Franke embeddings, Muckenhoupt weights, Sobolev spaces, Slobodetskii spaces, Bessel-potential spaces, Triebel-Lizorkin spaces, Besov spaces, vector-valued function spaces.

The second author was supported by a VIDI subsidy 639.032 .427 of the Netherlands Organisation for Scientific Research (NWO). 
Theorem 1.2. Let $w_{0}, w_{1} \in A_{\infty}, s_{0}>s_{1}, 0<p_{0} \leq p_{1}<\infty$ and $0<q_{0}, q_{1} \leq \infty$. Then there is a continuous embedding

if and only if

$$
F_{p_{0}, q_{0}}^{s_{0}}\left(\mathbb{R}^{d}, w_{0}\right) \hookrightarrow F_{p_{1}, q_{1}}^{s_{1}}\left(\mathbb{R}^{d}, w_{1}\right)
$$

$$
\sup _{\nu \in \mathbb{N}_{0}, m \in \mathbb{Z}^{d}} 2^{-\nu\left(s_{0}-s_{1}\right)} w_{0}\left(Q_{\nu, m}\right)^{-1 / p_{0}} w_{1}\left(Q_{\nu, m}\right)^{1 / p_{1}}<\infty
$$

In terms of the weighted means $\widetilde{w}(Q)=\frac{1}{|Q|} \int_{Q} w(x) d x$, the condition (C) reads

$$
\sup _{\nu \in \mathbb{N}_{0}, m \in \mathbb{Z}^{d}} 2^{-\nu\left[s_{0}-\frac{d}{p_{0}}-\left(s_{1}-\frac{d}{p_{1}}\right)\right]} \widetilde{w}_{0}\left(Q_{\nu, m}\right)^{-1 / p_{0}} \widetilde{w}_{1}\left(Q_{\nu, m}\right)^{1 / p_{1}}<\infty .
$$

In this way one immediately recovers the well-known inequality $s_{0}-\frac{d}{p_{0}} \geq s_{1}-\frac{d}{p_{1}}$ in the unweighted case $w_{0} \equiv w_{1} \equiv 1$.

Observe that the condition (C) differs from the one (1.1) for Besov spaces in its independence of the microscopic parameters $q_{0}, q_{1}$. It corresponds to (1.1) in case when $p_{*}=q_{*}=\infty$, i.e., $p_{0} \leq p_{1}$ and $q_{0} \leq q_{1}$. The independence of some properties of $F$-spaces - in particular sharp embeddings and trace spaces - of the microscopic parameters is well-known in the unweighted case [30. It is somewhat surprising and turns out to be very useful in the general vector-valued case, where $H$ - and $W$-spaces are not contained in the $F$ - and $B$-scale, see [19, 20, 26, 28. The special case of radial power weights $w(x)=|x|^{\gamma}$ was obtained in [18 based on a corresponding Plancherel-Pólya-Nikol'skii type inequality, which is interesting on its own.

As a consequence of Theorem 1.2 we may characterize various types of embeddings for vectorvalued spaces, at least in case of $A_{p}$-weights and $p_{0}>1$ (see Sections 3.3 and 3.4 for the proofs). In Section 4 we comment on the crucial condition (C) for some classes of power weights, see also [6, 12, and on the case $p_{1}<p_{0}$.

Theorem 1.3. Let $X$ be a Banach space, $s_{0}>s_{1}, 1<p_{0} \leq p_{1}<\infty, w_{0} \in A_{p_{0}}$ and $w_{1} \in A_{p_{1}}$. Then one has the continuous embeddings

$$
\begin{aligned}
& B_{p_{0}, q_{0}}^{s_{0}}\left(\mathbb{R}^{d}, w_{0} ; X\right) \hookrightarrow B_{p_{1}, q_{1}}^{s_{1}}\left(\mathbb{R}^{d}, w_{1} ; X\right), \\
& F_{p_{0}, q_{0}}^{s_{0}}\left(\mathbb{R}^{d}, w_{0} ; X\right) \hookrightarrow F_{p_{1}, q_{1}}^{s_{1}}\left(\mathbb{R}^{d}, w_{1} ; X\right), \\
& B_{p_{0}, p_{1}}^{s_{0}}\left(\mathbb{R}^{d}, w_{0} ; X\right) \hookrightarrow F_{p_{1}, q}^{s_{1}}\left(\mathbb{R}^{d}, w_{1} ; X\right), \\
& F_{p_{0}, q}^{s_{0}}\left(\mathbb{R}^{d}, w_{0} ; X\right) \hookrightarrow B_{p_{1}, p_{0}}^{s_{1}}\left(\mathbb{R}^{d}, w_{1} ; X\right), \\
& H^{s_{0}, p_{0}}\left(\mathbb{R}^{d}, w_{0} ; X\right) \hookrightarrow H^{s_{1}, p_{1}}\left(\mathbb{R}^{d}, w_{1} ; X\right) ; \\
& W^{s_{0}, p_{0}}\left(\mathbb{R}^{d}, w_{0} ; X\right) \hookrightarrow W^{s_{1}, p_{1}}\left(\mathbb{R}^{d}, w_{1} ; X\right), \\
& H^{s_{0}, p_{0}}\left(\mathbb{R}^{d}, w_{0} ; X\right) \hookrightarrow W^{s_{1}, p_{1}}\left(\mathbb{R}^{d}, w_{1} ; X\right), \\
& W^{s_{0}, p_{0}}\left(\mathbb{R}^{d}, w_{0} ; X\right) \hookrightarrow H^{s_{1}, p_{1}}\left(\mathbb{R}^{d}, w_{1} ; X\right), \\
& 1 \leq q_{0} \leq q_{1} \leq \infty \\
& 1 \leq q_{0}, q_{1} \leq \infty ; \\
& p_{0}<p_{1}, \quad 1 \leq q \leq \infty ; \\
& p_{0}<p_{1}, \quad 1 \leq q \leq \infty ; \\
& s_{0}>s_{1} \geq 0 ; \\
& s_{0}>s_{1} \geq 0 \text {; } \\
& s_{0}>0
\end{aligned}
$$

if and only if $(\mathbf{C})$ is satisfied.

The third and fourth embeddings, due to Jawerth and Franke [9, 14 in the unweighted setting, are based on real interpolation. They provide partial improvements of the embeddings for $F$-spaces from Theorem [1.2, in view of

$$
F_{p_{0}, p_{0}}^{s_{0}} \hookrightarrow B_{p_{0}, p_{1}}^{s_{0}}, \quad B_{p_{1}, p_{0}}^{s_{1}} \hookrightarrow F_{p_{1}, p_{1}}^{s_{1}} .
$$

We further record a consequence for the boundedness of the Bessel-potential operators, defined by Fourier transform, in the presence of weights. This gives a condition which might be easier to verify than the one in 22$]$.

Corollary 1.4. Let $X$ be a Banach space, $s>0,1<p_{0} \leq p_{1}<\infty, w_{0} \in A_{p_{0}}$ and $w_{1} \in A_{p_{1}}$. Then the operator $(1-\Delta)^{-s / 2}$ is bounded from $L^{p_{0}}\left(\mathbb{R}^{d}, w_{0} ; X\right)$ to $L^{p_{1}}\left(\mathbb{R}^{d}, w_{1} ; X\right)$ if and only if (C) is satisfied. 
In general, two-weight characterizations of bounded of linear operators can be difficult. Results in this direction for several standard operators (maximal operator, Hilbert Transform, Riesz potential, Bessel potential) can be found in [5, 13, 16, 17, 24, 25] and references therein. If one restricts to the class of Muckenhoupt weights, then characterizations are sometimes easier to obtain.

This paper is organized as follows. In Section 2 we introduce the function spaces and state their basic properties. The Theorems 1.2 and 1.3 are proved in Section 3 The validity of the condition (C) for some types of power weights is considered in Section 4 .

\section{FunCTION SPACES}

We summarize the basic properties of the function spaces under consideration. For details we refer to 2 , 3, 12, 18, 26, 30.

A locally integrable, almost everywhere positive function $w: \mathbb{R}^{d} \rightarrow[0, \infty)$ belongs to the Muckenhoupt class $A_{p}$ for some $1<p<\infty$, if

$$
[w]_{A_{p}}=\sup _{Q \text { cubes in } \mathbb{R}^{d}}\left(\frac{1}{|Q|} \int_{Q} w(x) d x\right)\left(\frac{1}{|Q|} \int_{Q} w(x)^{-\frac{1}{p-1}} d x\right)^{p-1}<\infty .
$$

One further defines $A_{\infty}=\bigcup_{1<p<\infty} A_{p}$. The Muckenhoupt classes are increasing in the sense that $A_{p_{0}} \subset A_{p_{1}}$ for $p_{0}<p_{1}$. For a Banach space $X, 0<p<\infty$ and $w \in A_{\infty}$ one sets

$$
\|f\|_{L^{p}\left(\mathbb{R}^{d}, w ; X\right)}=\left(\int_{\mathbb{R}^{d}}\|f(x)\|_{X}^{p} w(x) d x\right)^{1 / p} .
$$

This defines the spaces $L^{p}\left(\mathbb{R}^{d}, w ; X\right)$, which are Banach spaces for $p \geq 1$ and quasi-Banach spaces for $p<1$.

Let $\mathscr{S}\left(\mathbb{R}^{d} ; X\right)$ be space of $X$-valued Schwartz functions, let $\mathscr{S}^{\prime}\left(\mathbb{R}^{d} ; X\right)$ be the space of $X$-valued distributions, and let $\widehat{f}=\mathscr{F} f$ be the Fourier transform of $f \in \mathscr{S}^{\prime}\left(\mathbb{R}^{d} ; X\right)$. Choose a sequence $\left(\varphi_{k}\right)_{k \geq 0} \subset \mathscr{S}\left(\mathbb{R}^{d}\right)$ such that

$$
\widehat{\varphi}_{0}=\widehat{\varphi}, \quad \widehat{\varphi}_{1}(\xi)=\widehat{\varphi}(\xi / 2)-\widehat{\varphi}(\xi), \quad \widehat{\varphi}_{k}(\xi)=\widehat{\varphi}_{1}\left(2^{-k+1} \xi\right), \quad k \geq 2, \quad \xi \in \mathbb{R}^{d},
$$

with a generator function $\varphi$ of the form

$$
0 \leq \widehat{\varphi}(\xi) \leq 1, \quad \xi \in \mathbb{R}^{d}, \quad \widehat{\varphi}(\xi)=1 \quad \text { if }|\xi| \leq 1, \quad \widehat{\varphi}(\xi)=0 \text { if }|\xi| \geq \frac{3}{2} .
$$

Then equivalent (quasi-)norms for the Besov space $B$ and the Triebel-Lizorkin space $F$ are for $s \in \mathbb{R}, 0<p<\infty, 0<q \leq \infty, w \in A_{\infty}$ and $f \in \mathscr{S}^{\prime}\left(\mathbb{R}^{d} ; X\right)$ given by

$$
\begin{aligned}
& \|f\|_{B_{p, q}^{s}\left(\mathbb{R}^{d}, w ; X\right)}=\left\|\left(2^{s k} \varphi_{k} * f\right)_{k \geq 0}\right\|_{\ell^{q}\left(L^{p}\left(\mathbb{R}^{d}, w ; X\right)\right)}, \\
& \|f\|_{F_{p, q}^{s}\left(\mathbb{R}^{d}, w ; X\right)}=\left\|\left(2^{s k} \varphi_{k} * f\right)_{k \geq 0}\right\|_{L^{p}\left(\mathbb{R}^{d}, w ; \ell^{q}(X)\right)} .
\end{aligned}
$$

The $B$-spaces are defined in the same way also for $p=\infty$, the $F$-spaces require some more care in this case. For $s \in \mathbb{R}, 1<p<\infty$ and $w \in A_{p}$ the norm of the Bessel-potential space $H$ is given by

$$
\|f\|_{H^{s, p}\left(\mathbb{R}^{d}, w ; X\right)}=\left\|\mathscr{F}^{-1}\left[\left(1+|\cdot|^{2}\right)^{s / 2} \widehat{f}\right]\right\|_{L^{p}\left(\mathbb{R}^{d}, w ; X\right)} .
$$

For $m \in \mathbb{N}_{0}, 1<p<\infty$ and $w \in A_{p}$ the norm of the classical Sobolev space $W$ is

$$
\|f\|_{W^{m, p}\left(\mathbb{R}^{d}, w ; X\right)}=\left(\sum_{|\alpha| \leq m}\left\|D^{\alpha} f\right\|_{L^{p}\left(\mathbb{R}^{d}, w ; X\right)}^{p}\right)^{1 / p} .
$$

For $s \geq 0$ one defines the Sobolev-Slobodetskii scale by

$$
W^{s, p}\left(\mathbb{R}^{d}, w ; X\right)= \begin{cases}W^{m, p}\left(\mathbb{R}^{d}, w ; X\right), & s=m \in \mathbb{N}_{0}, \\ B_{p, p}^{s}\left(\mathbb{R}^{d}, w ; X\right)=F_{p, p}^{s}\left(\mathbb{R}^{d}, w ; X\right), & s \notin \mathbb{N}_{0} .\end{cases}
$$

In the scalar case we write $L^{p}\left(\mathbb{R}^{d}, w\right)=L^{p}\left(\mathbb{R}^{d}, w ; \mathbb{C}\right)$, and so on.

We note some basic relations. Monotonicity of the $\ell^{q}$ spaces implies that for $0<q_{0} \leq q_{1} \leq \infty$

$$
B_{p, q_{0}}^{s}\left(\mathbb{R}^{d}, w ; X\right) \hookrightarrow B_{p, q_{1}}^{s}\left(\mathbb{R}^{d}, w ; X\right), \quad F_{p, q_{0}}^{s}\left(\mathbb{R}^{d}, w ; X\right) \hookrightarrow F_{p, q_{1}}^{s}\left(\mathbb{R}^{d}, w ; X\right) .
$$


For $\varepsilon>0$ and arbitrary $0<q_{0}, q_{1} \leq \infty$ one has

$$
B_{p, q_{0}}^{s+\varepsilon}\left(\mathbb{R}^{d}, w ; X\right) \hookrightarrow B_{p, q_{1}}^{s}\left(\mathbb{R}^{d}, w ; X\right), \quad F_{p, q_{0}}^{s+\varepsilon}\left(\mathbb{R}^{d}, w ; X\right) \hookrightarrow F_{p, q_{1}}^{s}\left(\mathbb{R}^{d}, w ; X\right),
$$

and for $0<p<\infty$, by Minkowski's inequality,

$$
B_{p, \min \{p, q\}}^{s}\left(\mathbb{R}^{d}, w ; X\right) \hookrightarrow F_{p, q}^{s}\left(\mathbb{R}^{d}, w ; X\right) \hookrightarrow B_{p, \max \{p, q\}}^{s}\left(\mathbb{R}^{d}, w ; X\right) .
$$

The $H$-spaces are related to the $F$-spaces as follows. In the scalar case one has

$$
H^{s, p}\left(\mathbb{R}^{d}, w\right)=F_{p, 2}^{s}\left(\mathbb{R}^{d}, w\right), \quad 1<p<\infty, \quad w \in A_{p} .
$$

In the vector-valued case this is true if and only if $X$ can be renormed as a Hilbert space. One further has $W^{m, p}\left(\mathbb{R}^{d} ; X\right)=H^{m, p}\left(\mathbb{R}^{d} ; X\right)$ for $m \in \mathbb{N}$ if and only if $X$ is a UMD Banach space. For general Banach spaces $X$ one still has

$$
\begin{gathered}
F_{p, 1}^{s}\left(\mathbb{R}^{d}, w ; X\right) \hookrightarrow H^{s, p}\left(\mathbb{R}^{d}, w ; X\right) \hookrightarrow F_{p, \infty}^{s}\left(\mathbb{R}^{d}, w ; X\right), \quad 1<p<\infty, \quad w \in A_{p} . \\
F_{p, 1}^{s}\left(\mathbb{R}^{d}, w ; X\right) \hookrightarrow W^{s, p}\left(\mathbb{R}^{d}, w ; X\right) \hookrightarrow F_{p, \infty}^{s}\left(\mathbb{R}^{d}, w ; X\right), \quad s \geq 0, \quad 1<p<\infty, \quad w \in A_{p} .
\end{gathered}
$$

For the latter embeddings in (2.6) and (2.7) to hold, a local $A_{p}$-condition is in fact necessary, see [18, Remark 3.13] and [23. The embeddings can be improved with respect to the microscopic parameters if one takes into account type and cotype of $X$, see [31, Proposition 3.1] and [19, Proposition 5.8].

\section{Proofs of Theorems 1.2 and 1.3}

After a few preparations on products of weights, in Section 3.2 we prove Theorem 1.2 , The scalar version of the Jawerth-Franke type embeddings from Theorem 1.3 is derived in Section 3.3 . The remaining assertions and the vector-valued version of Theorem 1.3 are shown in Section 3.4 .

3.1. Preparations. The following assertions on products of Muckenhoupt weights will be needed.

Lemma 3.1. Let $1<p<\infty$ and $w_{0}, w_{1} \in A_{p}$. Then there is $\eta_{0}>0$ such that for all $\varepsilon, \delta \in\left[0, \eta_{0}\right)$ one has $w_{0}^{-\varepsilon} w_{1}^{1+\delta} \in A_{p}$.

Proof. By [11, Proposition 9.1.5] one has $w_{0}^{-1 /(p-1)} \in A_{p^{\prime}}$, such that $w_{0}^{-\tau_{0}} \in A_{p}$ for sufficiently small $\tau_{0}>0$ by [11, Exercise 9.1.3]. Furthermore, [11, Theorem 9.2.5] shows that there is $\gamma_{0}>0$ such that $w_{1}^{1+\gamma_{0}} \in A_{p}$. Hence, again by [11, Exercise 9.1.3], we have $w_{0}^{-\tau_{0} \theta}, w_{1}^{\left(1+\gamma_{0}\right) \lambda} \in A_{p}$ for all $\theta, \lambda \in[0,1]$. Using [11, Exercise 9.1.10], this implies

$$
w_{0}^{-\tau_{0} \theta(1-t)} w_{1}^{\left(1+\gamma_{0}\right) \lambda t} \in A_{p}, \quad \theta, \lambda, t \in[0,1] .
$$

Letting $t=\frac{2}{2+\gamma_{0}}$ and $\sigma_{0}=\frac{\gamma_{0}}{2+\gamma_{0}}$, this gives $w_{0}^{-\tau_{0} \sigma_{0} \theta} w_{1}^{\left(1+\sigma_{0}\right) \lambda} \in A_{p}$ for all $\theta, \lambda \in[0,1]$, which implies the assertion.

Lemma 3.2. Let $w_{0}, w_{1} \in A_{\infty}$. Then there are $\eta_{0}>0$ and a constant $C>0$ such that for all $\varepsilon, \delta \in\left(0, \eta_{0}\right)$ and all d-dimensional cubes $Q \subset \mathbb{R}^{d}$ we have

$$
\int_{Q} w_{0}^{-\varepsilon} w_{1}^{1+\delta} d x \leq C|Q|^{\varepsilon-\delta}\left(\int_{Q} w_{0} d x\right)^{-\varepsilon}\left(\int_{Q} w_{1} d x\right)^{1+\delta} .
$$

Proof. Fix $p>1$ such that $w_{0}, w_{1} \in A_{p}$. Choose $\eta_{0}>0$ such that $\frac{1}{\eta_{0}(p-1)}>1$, and let $\varepsilon \in\left(0, \eta_{0}\right)$. Then for all $\delta>0$, Hölder's inequality with $q=\frac{1}{\varepsilon(p-1)}$ and $q^{\prime}=\frac{1}{1-\varepsilon(p-1)}$ yields

$$
\int_{Q} w_{0}^{-\varepsilon} w_{1}^{1+\delta} d x \leq\left(\int_{Q} w_{0}^{-\frac{1}{p-1}} d x\right)^{\varepsilon(p-1)}\left(\int_{Q} w_{1}^{\frac{1+\delta}{1-\varepsilon(p-1)}} d x\right)^{1-\varepsilon(p-1)}
$$

By definition of $\left[w_{0}\right]_{A_{p}}$,

$$
\left(\int_{Q} w_{0}^{-\frac{1}{p-1}} d x\right)^{\varepsilon(p-1)} \leq\left[w_{0}\right]_{A_{p}}^{\varepsilon}|Q|^{\varepsilon p}\left(\int_{Q} w_{0} d x\right)^{-\varepsilon} .
$$


The reverse Hölder inequality [11, Theorem 9.2.2] implies that there are $\gamma_{0}>0$ and $C>0$, independent of $Q$, such that for all $\gamma \in\left(0, \gamma_{0}\right]$ we have

$$
\int_{Q} w_{1}^{1+\gamma} d x \leq C|Q|^{-\gamma}\left(\int_{Q} w_{1} d x\right)^{1+\gamma}
$$

Decreasing $\eta_{0}$ if necessary, we may assume that $\frac{1+\eta_{0}}{1-\eta_{0}(p-1)} \leq 1+\gamma_{0}$. For $\varepsilon, \delta \in\left(0, \eta_{0}\right)$ we then obtain

$$
\left(\int_{Q} w_{1}^{\frac{1+\delta}{1-\varepsilon(p-1)}} d x\right)^{1-\varepsilon(p-1)} \leq C|Q|^{-\varepsilon(p-1)-\delta}\left(\int_{Q} w_{1} d x\right)^{1+\delta} .
$$

Applying the estimates (3.2) and (3.3) in (3.1), the assertion follows.

The independence of the microscopic parameters in Theorem 1.2 is essentially a consequence of the following Gagliardo-Nirenberg type inequality, see [1, 21, 28] for the unweighted setting and [18, Proposition 5.1] for the weighted setting.

Proposition 3.3. Let $X$ be a Banach space, $s_{0}>s_{1}, 0<p_{0}, p_{1}<\infty$ and $w_{0}, w_{1} \in A_{\infty}$. Given $\theta \in(0,1)$, let $s_{1}<s<s_{0}, 0<p<\infty$ and $w \in A_{\infty}$ be defined by

$$
s=(1-\theta) s_{0}+\theta s_{1}, \quad \frac{1}{p}=\frac{1-\theta}{p_{0}}+\frac{\theta}{p_{1}}, \quad w=w_{0}^{(1-\theta) p / p_{0}} w_{1}^{\theta p / p_{1}} .
$$

Then for arbitrary $0<q, q_{0}, q_{1} \leq \infty$ there is a constant $C>0$ such that for all $f \in \mathscr{S}^{\prime}\left(\mathbb{R}^{d} ; X\right)$ one has

$$
\|f\|_{F_{p, q}^{s}\left(\mathbb{R}^{d}, w ; X\right)} \leq C\|f\|_{F_{p_{0}, q_{0}}^{s_{0}}\left(\mathbb{R}^{d}, w_{0} ; X\right)}^{1-\theta}\|f\|_{F_{p_{1}, q_{1}}^{s_{1}}\left(\mathbb{R}^{d}, w_{1} ; X\right)}^{\theta} .
$$

Note that in [18] the case $0<\min \left\{p, p_{0}, p_{1}, q, q_{0}, q_{1}\right\} \leq 1$ has not been considered. However, the proof extends to this case.

3.2. Proof of Theorem 1.2. Necessity. Assume $F_{p_{0}, q_{0}}^{s_{0}}\left(\mathbb{R}^{d}, w_{0}\right) \hookrightarrow F_{p_{1}, q_{1}}^{s_{1}}\left(\mathbb{R}^{d}, w_{1}\right)$. Then (2.4) implies that for $r_{0}=\min \left\{p_{0}, q_{0}\right\}$ and $r_{1}=\max \left\{p_{1}, q_{1}\right\}$ we have

$$
B_{p_{0}, r_{0}}^{s_{0}}\left(\mathbb{R}^{d}, w_{0}\right) \hookrightarrow F_{p_{0}, q_{0}}^{s_{0}}\left(\mathbb{R}^{d}, w_{0}\right) \hookrightarrow F_{p_{1}, q_{1}}^{s_{1}}\left(\mathbb{R}^{d}, w_{1}\right) \hookrightarrow B_{p_{1}, r_{1}}^{s_{1}}\left(\mathbb{R}^{d}, w_{1}\right) .
$$

Hence Proposition 1.1 and the assumption $p_{0} \leq p_{1}$ show that (C) is satisfied.

Sufficiency. Assuming (C), we prove

$$
F_{p_{0}, q_{0}}^{s_{0}}\left(\mathbb{R}^{d}, w_{0}\right) \hookrightarrow F_{p_{1}, q_{1}}^{s_{1}}\left(\mathbb{R}^{d}, w_{1}\right)
$$

Observe that by (2.3), it suffices to consider the case $q_{1} \leq \min \left\{p_{0}, p_{1}\right\}$, which we will assume in the sequel.

Since $p_{1} \geq p_{0}$, there is $\theta_{0} \in[0,1)$ such that $\frac{1}{p_{1}}-\frac{1-\theta_{0}}{p_{0}}=0$. For $\theta \in\left(\theta_{0}, 1\right)$ we set

$$
\varepsilon=\frac{\frac{1-\theta}{p_{0}}}{\frac{1}{p_{1}}-\frac{1-\theta}{p_{0}}}>0 .
$$

Observe that $\varepsilon \searrow 0$ as $\theta \nearrow 1$. Define the weight $v$ and the numbers $r$ and $t$ by

$$
v=w_{0}^{-\varepsilon} w_{1}^{1+\varepsilon}, \quad \frac{1}{p_{1}}=\frac{1-\theta}{p_{0}}+\frac{\theta}{r}, \quad s_{1}=(1-\theta) s_{0}+\theta t .
$$

Then one can check that

$$
w_{1}=w_{0}^{(1-\theta) p_{1} / p_{0}} v^{p_{1} \theta / r}, \quad r \in\left[p_{1}, \infty\right), \quad t<s_{1}<s_{0} .
$$

Moreover, $v \in A_{p_{1}} \subset A_{r}$ if $\theta$ is sufficiently close to 1 by Lemma 3.1. Now let $f \in \mathscr{S}^{\prime}\left(\mathbb{R}^{d}\right)$. From Proposition 3.3 we infer the Gagliardo-Nirenberg inequality

$$
\|f\|_{F_{p_{1}, q_{1}}^{s_{1}}\left(\mathbb{R}^{d}, w_{1}\right)} \leq C\|f\|_{F_{p_{0}, q_{0}}^{s_{0}}\left(\mathbb{R}^{d}, w_{0}\right)}^{1-\theta}\|f\|_{F_{r, r}^{t}\left(\mathbb{R}^{d}, v\right)}^{\theta}
$$

We now claim that for $\theta$ sufficiently close to 1 we have

$$
\|f\|_{F_{r, r}^{t}\left(\mathbb{R}^{d}, v\right)} \leq C\|f\|_{F_{p_{1}, p_{1}}^{s_{1}}\left(\mathbb{R}^{d}, w_{1}\right)} .
$$


Since $B_{p, p}^{s}=F_{p, p}^{s}$ and $r \geq p_{1}$, by Proposition 1.1 this is true if and only if

$$
\sup _{\nu \in \mathbb{N}_{0}, m \in \mathbb{Z}^{d}} 2^{-\nu\left(s_{1}-t\right)} w_{1}\left(Q_{\nu, m}\right)^{-1 / p_{1}} v\left(Q_{\nu, m}\right)^{1 / r}<\infty .
$$

To check this condition, we note that since $v=w_{0}^{-\varepsilon} w_{1}^{1+\varepsilon}$, Lemma 3.2 implies

$$
v\left(Q_{\nu, m}\right) \leq C w_{0}\left(Q_{\nu, m}\right)^{-\varepsilon} w_{1}\left(Q_{\nu, m}\right)^{1+\varepsilon}
$$

provided $\varepsilon$ is sufficiently small. Using the relations $s_{1}-t=\left(s_{0}-s_{1}\right) \frac{1-\theta}{\theta}, \frac{\varepsilon}{r}=\frac{1}{p_{0}} \frac{1-\theta}{\theta}$ and $\frac{1+\varepsilon}{r}=\frac{1}{\theta p_{1}}$, it follows that

$$
2^{-\nu\left(s_{1}-t\right)} v\left(Q_{\nu, m}\right)^{1 / r} w_{1}\left(Q_{\nu, m}\right)^{-1 / p_{1}} \leq C\left(2^{-\nu\left(s_{0}-s_{1}\right)} w_{0}\left(Q_{\nu, m}\right)^{-1 / p_{0}} w_{1}\left(Q_{\nu, m}\right)^{1 / p_{1}}\right)^{\frac{1-\theta}{\theta}} .
$$

The latter is uniformly bounded in $\nu$ and $m$ by the assumption (C). This yields (3.6). Applying (3.6) in (3.5), we find

$$
\|f\|_{F_{p_{1}, q_{1}}^{s_{1}}\left(\mathbb{R}^{d}, w_{1}\right)} \leq C\|f\|_{F_{p_{0}, q_{0}}^{s_{0}}\left(\mathbb{R}^{d}, w_{0}\right)}^{1-\theta}\|f\|_{F_{p_{1}, p_{1}}^{s_{1}}\left(\mathbb{R}^{d}, w_{1}\right)}^{\theta} .
$$

Since $q_{1} \leq p_{1}$ by the above assumption, on the right-hand side we may replace $F_{p_{1}, p_{1}}^{s_{1}}$ by $F_{p_{1}, q_{1}}^{s_{1}}$ and then divide by $\|f\|_{F_{p_{1}, q_{1}}^{s_{1}}\left(\mathbb{R}^{d}, w_{1}\right)}^{\theta}$, which implies

$$
\|f\|_{F_{p_{1}, q_{1}}^{s_{1}}\left(\mathbb{R}^{d}, w_{1}\right)} \leq C\|f\|_{F_{p_{0}, q_{0}}^{s_{0}}\left(\mathbb{R}^{d}, w_{0}\right)}, \quad f \in \mathbb{Y}:=F_{p_{0}, q_{0}}^{s_{0}}\left(\mathbb{R}^{d}, w_{0}\right) \cap F_{p_{1}, q_{1}}^{s_{1}}\left(\mathbb{R}^{d}, w_{1}\right) .
$$

The extension from $f \in \mathbb{Y}$ to all $f \in F_{p_{0}, q_{0}}^{s_{0}}\left(\mathbb{R}^{d}, w_{0}\right)$ is a matter of approximation. In case $q_{0}<\infty$, the embedding (3.4) follows from the density of the Schwartz class in $F_{p_{0}, q_{0}}^{s_{0}}\left(\mathbb{R}^{d}, w_{0}\right)$. For $q_{0}=\infty$, we observe that $B_{p_{0}, q_{1}}^{s_{0}}\left(\mathbb{R}^{d}, w_{0}\right) \hookrightarrow \mathbb{Y}$, employing elementary embeddings and (C) to apply Proposition 1.1. For $f \in F_{p_{0}, \infty}^{s_{0}}\left(\mathbb{R}^{d}, w_{0}\right)$ we set $f_{n}=\sum_{k=0}^{n} \varphi_{n} * f$. Then $f_{n} \rightarrow f$ in $\mathscr{S}^{\prime}\left(\mathbb{R}^{d}\right)$ as $n \rightarrow \infty, f_{n} \in B_{p_{0}, q_{1}}^{s_{0}}\left(\mathbb{R}^{d}, w_{0}\right) \hookrightarrow \mathbb{Y}$ for all $n$ and

$$
\left\|f_{n}\right\|_{F_{p_{1}, q_{1}}^{s_{1}}\left(\mathbb{R}^{d}, w_{1}\right)} \leq C\left\|f_{n}\right\|_{F_{p_{0}, \infty}^{s_{0}}\left(\mathbb{R}^{d}, w_{0}\right)} \leq C\|f\|_{F_{p_{0}, \infty}^{s_{0}}\left(\mathbb{R}^{d}, w_{0}\right)}
$$

using [18, Proposition 2.4] for the latter inequality. Now the Fatou property of $F_{p_{1}, q_{1}}^{s_{1}}\left(\mathbb{R}^{d}, w_{1}\right)$, see [26. Proposition 2.18], implies that $f \in F_{p_{1}, q_{1}}^{s_{1}}\left(\mathbb{R}^{d}, w_{1}\right)$ and that the asserted estimate holds for $f$. This completes the proof of (3.4).

3.3. Proof of Jawerth-Franke embeddings in the scalar case. Next we show embeddings of Jawerth-Franke type for scalar-valued spaces, i.e., the third and fourth embeddings in Theorem 1.3 for $X=\mathbb{C}$. We argue similar as in [18, Theorem 6.2] and [27, Theorem 6].

Theorem 3.4. Assume $s_{0}>s_{1}, 1<p_{0}<p_{1}<\infty, q \in[1, \infty], w_{0} \in A_{p_{0}}$ and $w_{1} \in A_{p_{1}}$. Then the embeddings

$$
\begin{gathered}
B_{p_{0}, p_{1}}^{s_{0}}\left(\mathbb{R}^{d}, w_{0}\right) \hookrightarrow F_{p_{1}, q}^{s_{1}}\left(\mathbb{R}^{d}, w_{1}\right), \\
F_{p_{0}, q}^{s_{0}}\left(\mathbb{R}^{d}, w_{0}\right) \hookrightarrow B_{p_{1}, p_{0}}^{s_{1}}\left(\mathbb{R}^{d}, w_{1}\right)
\end{gathered}
$$

are continuous if and only if $(\mathbf{C})$ is satisfied.

Proof. Necessity. Assume that one of the embeddings is continuous. By (2.2),

$$
F_{p_{0}, p_{0}}^{s_{0}}\left(\mathbb{R}^{d}, w_{0}\right) \hookrightarrow B_{p_{0}, p_{1}}^{s_{0}}\left(\mathbb{R}^{d}, w_{0}\right) \text { and } B_{p_{1}, p_{0}}^{s_{1}}\left(\mathbb{R}^{d}, w_{1}\right) \hookrightarrow F_{p_{1}, p_{1}}^{s_{1}}\left(\mathbb{R}^{d}, w_{1}\right),
$$

which then implies an embedding for $F$-spaces as in Theorem 1.2 , This is equivalent to (C).

Sufficiency. Assume (C) is satisfied.

Step 1. We prove (3.7). It suffices to consider the case $q=1$ by (2.2). Let $r_{0}, r_{1} \in(1, \infty)$ be such that $p_{0}<r_{0}<p_{1}<r_{1}$, and define $\theta \in(0,1)$ by

$$
\frac{1}{p_{1}}=\frac{1-\theta}{r_{0}}+\frac{\theta}{r_{1}} \text {. }
$$

Let $a \in(0,1)$ be a small parameter and $b=\frac{(1-\theta) a}{\theta}>0$. Set $t_{0}=s_{0}-a\left(s_{0}-s_{1}\right)$ and $t_{1}=$ $s_{0}+b\left(s_{0}-s_{1}\right)$. Then one can check that $(1-\theta) t_{0}+\theta t_{1}=s_{0}$ and $s_{1}<t_{0}<s_{0}<t_{1}$. Now define the weights $v_{0}$ and $v_{1}$ by

$$
v_{0}^{\frac{1}{r_{0}}}=w_{0}^{\frac{a}{p_{0}}} w_{1}^{\frac{1-a}{p_{1}}}, \quad v_{1}^{\frac{1}{r_{1}}}=w_{0}^{-\frac{b}{p_{0}}} w_{1}^{\frac{1+b}{p_{1}}}
$$


Then $v_{0} \in A_{r_{0}}$ by [11, Exercise 9.1.5]. Choosing $r_{1}$ close to $p_{1}$ and $a$ small, such that $b$ becomes small, we further have $v_{1} \in A_{r_{1}}$ by Lemma 3.1. After these preparations we can argue in the usual way. By [18, Proposition 6.1] one has

$$
B_{p_{0}, p_{1}}^{s_{0}}\left(\mathbb{R}^{d}, w_{0}\right)=\left(F_{p_{0}, 1}^{t_{0}}\left(\mathbb{R}^{d}, w_{0}\right), F_{p_{0}, 1}^{t_{1}}\left(\mathbb{R}^{d}, w_{0}\right)\right)_{\theta, p_{1}}
$$

where $(\cdot, \cdot)_{\theta, p_{1}}$ denotes the real interpolation functor. We claim that

$$
F_{p_{0}, 1}^{t_{0}}\left(\mathbb{R}^{d}, w_{0}\right) \hookrightarrow F_{r_{0}, 1}^{s_{1}}\left(\mathbb{R}^{d}, v_{0}\right), \quad F_{p_{0}, 1}^{t_{1}}\left(\mathbb{R}^{d}, w_{0}\right) \hookrightarrow F_{r_{1}, 1}^{s_{1}}\left(\mathbb{R}^{d}, v_{1}\right) .
$$

In fact, by the above choices, for $\nu \in \mathbb{N}_{0}$ and $m \in \mathbb{Z}^{d}$ we have

$$
2^{-\nu\left(t_{0}-s_{1}\right)} w_{0}\left(Q_{\nu, m}\right)^{-1 / p_{0}} v_{0}\left(Q_{\nu, m}\right)^{1 / r_{0}}=\left(2^{-\nu\left(s_{0}-s_{1}\right)} w_{0}\left(Q_{\nu, m}\right)^{-1 / p_{0}} w_{1}\left(Q_{\nu, m}\right)^{1 / p_{1}}\right)^{1-a} .
$$

Here, the right-hand side is bounded in $\nu$ and $m$ by assumption (C). Hence Theorem 1.2 yields the first embedding in (3.9). The second embedding follows in the same way. We therefore find

$$
B_{p_{0}, p_{1}}^{s_{0}}\left(\mathbb{R}^{d}, w_{0}\right) \hookrightarrow\left(F_{r_{0}, 1}^{s_{1}}\left(\mathbb{R}^{d}, v_{0}\right), F_{r_{1}, 1}^{s_{1}}\left(\mathbb{R}^{d}, v_{1}\right)\right)_{\theta, p_{1}} .
$$

Since $\frac{1}{p_{1}}=\frac{1-\theta}{r_{0}}+\frac{\theta}{r_{1}}$ and $w_{1}^{\frac{1}{p_{1}}}=v_{0}^{\frac{1-\theta}{r_{0}}} v_{1}^{\frac{\theta}{r_{1}}}$, [18, Proposition 6.1] shows that the latter interpolation space embeds into $F_{p_{1}, 1}^{s_{1}}\left(\mathbb{R}^{d}, w_{1}\right)$. This proves (3.7).

Step 2. We derive (3.8) in a similar way. Here it suffices to consider $q=\infty$. Let $r_{0}, r_{1} \in(1, \infty)$ be such that $r_{0}<p_{0}<r_{1}<p_{1}$. Let $\theta \in(0,1)$ be such that

$$
\frac{1}{p_{0}}=\frac{1-\theta}{r_{0}}+\frac{\theta}{r_{1}} \text {. }
$$

Let $b \in(0,1)$ be small and $a=\frac{\theta b}{1-\theta}$. Set $t_{0}=s_{1}-a\left(s_{0}-s_{1}\right)$ and $t_{1}=s_{1}+b\left(s_{0}-s_{1}\right)$, such that $(1-\theta) t_{0}+\theta t_{1}=s_{1}$ and $t_{0}<s_{1}<t_{1}<s_{0}$. Define the weights $v_{0}$ and $v_{1}$ by

$$
v_{0}^{\frac{1}{r_{0}}}=w_{0}^{\frac{1+a}{p_{0}}} w_{1}^{-\frac{a}{p_{1}}}, \quad v_{1}^{\frac{1}{r_{1}}}=w_{0}^{\frac{1-b}{p_{0}}} w_{1}^{\frac{b}{p_{1}}}
$$

Choosing $r_{0}$ close to $p_{0}$ and $b$ small, as above we obtain $v_{0} \in A_{r_{0}}$, and further $v_{1} \in A_{r_{1}}$. The fact that $w_{0}^{\frac{1}{p_{0}}}=v_{0}^{\frac{1-\theta}{r_{0}}} v_{1}^{\frac{\theta}{r_{1}}}$ allows to apply [18, Proposition 6.1] to the result

$$
F_{p_{0}, \infty}^{s_{0}}\left(\mathbb{R}^{d}, w_{0}\right)=\left(F_{r_{0}, \infty}^{s_{0}}\left(\mathbb{R}^{d}, v_{0}\right), F_{r_{1}, \infty}^{s_{0}}\left(\mathbb{R}^{d}, v_{1}\right)\right)_{\theta, p_{0}}
$$

By (2.4) and Proposition 1.1 we find

$$
\begin{aligned}
& F_{r_{0}, \infty}^{s_{0}}\left(\mathbb{R}^{d}, v_{0}\right) \hookrightarrow B_{r_{0}, \infty}^{s_{0}}\left(\mathbb{R}^{d}, v_{0}\right) \hookrightarrow B_{p_{1}, \infty}^{t_{0}}\left(\mathbb{R}^{d}, w_{1}\right), \\
& F_{r_{1}, \infty}^{s_{0}}\left(\mathbb{R}^{d}, v_{1}\right) \hookrightarrow B_{r_{1}, \infty}^{s_{0}}\left(\mathbb{R}^{d}, v_{1}\right) \hookrightarrow B_{p_{1}, \infty}^{t_{1}}\left(\mathbb{R}^{d}, w_{1}\right),
\end{aligned}
$$

where the conditions for the latter Sobolev embeddings are satisfied by the above choices and the assumption $(\mathbf{C})$. Applying these and interpolating once more we obtain

$$
F_{p_{0}, \infty}^{s_{0}}\left(\mathbb{R}^{d}, w_{0}\right) \hookrightarrow\left(B_{p_{1}, \infty}^{t_{0}}\left(\mathbb{R}^{d}, w_{1}\right), B_{p_{1}, \infty}^{t_{1}}\left(\mathbb{R}^{d}, w_{1}\right)\right)_{\theta, p_{0}}=B_{p_{1}, p_{0}}^{s_{1}}\left(\mathbb{R}^{d}, w_{1}\right)
$$

This shows (3.8).

Remark 3.5. Although we only presented Theorem 3.4 in the case $p_{0}, p_{1}>1, q \in[1, \infty], w_{0} \in A_{p_{0}}$ and $w_{1} \in A_{p_{1}}$, parts of the result can directly be extended to the full parameter range and weights in $A_{\infty}$. However, it seems that for the general result the required interpolation identities are not available in the literature, see the open problems stated in [2], and [18, Remark 6.6]. 
3.4. Proof of Theorem 1.3. Let $X$ be an arbitrary Banach space. From the proof in 12 and references therein it seems that Proposition 1.1 and thus Theorem 1.2 remain true in the $X$-valued setting. However, it is quite some work to check all this. Restricting to important special cases, based on the positivity of the Bessel-potential operators we can give a short argument to derive the vector-valued case directly from the scalar case.

Necessity. Assume that in Theorem 1.3 any of the asserted embeddings holds. Then it in particular holds in the scalar case $X=\mathbb{C}$. For the first four embeddings we have already seen that $(\mathbf{C})$ is necessary. For the latter four embeddings, we note that by (2.2), (2.4), (2.5) and (2.7) one has

$$
F_{p_{0}, 1}^{s_{0}} \hookrightarrow H^{s_{0}, p_{0}}, W^{s_{0}, p_{0}} ; \quad H^{s_{1}, p_{1}}, W^{s_{1}, p_{1}} \hookrightarrow F_{p_{1}, \infty}^{s_{1}}
$$

Hence any of the latter four embeddings implies $F_{p_{0}, 1}^{s_{0}}\left(\mathbb{R}^{d}, w_{0}\right) \hookrightarrow F_{p_{1}, \infty}^{s_{1}}\left(\mathbb{R}^{d}, w_{1}\right)$, for which $(\mathbf{C})$ is necessary by Theorem 1.2 .

Sufficiency. Assuming (C), we show the embeddings stated in Theorem 1.3. We know from Theorem 1.2 and (2.5) that

$$
H^{s_{0}, p_{0}}\left(\mathbb{R}^{d}, w_{0}\right) \hookrightarrow H^{s_{1}, p_{1}}\left(\mathbb{R}^{d}, w_{1}\right) .
$$

This is equivalent to the continuity of the Bessel-potential operator $(1-\Delta)^{-\frac{s_{0}-s_{1}}{2}}$ from $L^{p_{0}}\left(\mathbb{R}^{d}, w_{0}\right)$ to $L^{p_{1}}\left(\mathbb{R}^{d}, w_{1}\right)$. Since $s_{0} \geq s_{1}$, this operator is positive, see [11, Proposition 6.1.5]. Hence, by [10, Theorem V.1.12], it extends continuously to an operator from $L^{p_{0}}\left(\mathbb{R}^{d}, w_{0} ; X\right)$ to $L^{p_{1}}\left(\mathbb{R}^{d}, w_{1} ; X\right)$, for arbitrary $X$. This implies

$$
H^{s_{0}, p_{0}}\left(\mathbb{R}^{d}, w_{0} ; X\right) \hookrightarrow H^{s_{1}, p_{1}}\left(\mathbb{R}^{d}, w_{1} ; X\right) .
$$

Now real interpolation, see [18, Proposition 6.1], shows the asserted embedding for $X$-valued $B$ spaces. Repeating literally the proofs of Theorems 1.2 and 3.4 , we may altogether deduce the first five embeddings of Theorem 1.3 in the vector-valued case. The remaining three embeddings for $H$ - and $W$-spaces are now a consequence of

$$
H^{s_{0}, p_{0}}, W^{s_{0}, p_{0}} \hookrightarrow F_{p_{0}, \infty}^{s_{0}} ; \quad F_{p_{1}, 1}^{s_{1}} \hookrightarrow H^{s_{1}, p_{1}}, W^{s_{1}, p_{1}},
$$

see (2.2), (2.6) and (2.7), and the embedding $F_{p_{0}, \infty}^{s_{0}}\left(\mathbb{R}^{d}, w_{0} ; X\right) \hookrightarrow F_{p_{1}, 1}^{s_{1}}\left(\mathbb{R}^{d}, w_{1} ; X\right)$.

\section{Power Weights}

In this section we consider the crucial condition $(\mathbf{C})$, which characterizes the validity of the embeddings in Theorems 1.2 and 1.3 , for some classes of power weights $w_{0}$ and $w_{1}$. See also 6, 12.

Throughout, we fix the parameters $s_{0}>s_{1}$ and $p_{0} \leq p_{1}$.

4.1. Radial power weights. For $\alpha, \beta>-d$, let $w_{\alpha, \beta} \in A_{\infty}$ be defined by

$$
w_{\alpha, \beta}(x)= \begin{cases}|x|^{\alpha} & \text { if }|x| \leq 1 \\ |x|^{\beta} & \text { if }|x|>1\end{cases}
$$

One has $w_{\alpha, \beta} \in A_{p}$ if and only if $\alpha, \beta \in(-d, d(p-1))$, see [12, Proposition 2.6]. As in [12, Proposition 2.8], we have the following characterization.

Proposition 4.1. Let $\alpha_{0}, \beta_{0}, \alpha_{1}, \beta_{1}>-d$. Then the weights

$$
w_{0}=w_{\alpha_{0}, \beta_{0}}, \quad w_{1}=w_{\alpha_{1}, \beta_{1}}
$$

satisfy (C) if and only if

$$
s_{0}-\frac{d+\alpha_{0}}{p_{0}} \geq s_{1}-\frac{d+\alpha_{1}}{p_{1}}, \quad s_{0}-\frac{d}{p_{0}} \geq s_{1}-\frac{d}{p_{1}}, \quad \frac{\beta_{0}}{p_{0}} \geq \frac{\beta_{1}}{p_{1}} .
$$

Observe that these three conditions correspond to a condition at $x=0$, where the powers $\alpha_{0}, \alpha_{1}$ are relevant, for $x$ away from zero and infinity, where the spaces are essentially unweighted, and at infinity, where $\beta_{0}, \beta_{1}$ are relevant.

Let us give the details how to derive (4.2). For $\gamma>-d, \nu \in \mathbb{N}_{0}$ and $m \in \mathbb{Z}^{d}$ we note that

$$
w_{\gamma, \gamma}\left(Q_{\nu, 0}\right) \sim 2^{-\nu(d+\gamma)}, \quad w_{\gamma, \gamma}\left(Q_{\nu, m}\right) \sim 2^{-\nu(d+\gamma)}|m|^{\gamma} \quad(m \neq 0) .
$$


Recall here that a cube $Q_{\nu, m}$ is centered at $2^{-\nu} m$ and has side length $2^{-\nu}$. We need to distinguish the following cases. For the indices $I_{1}=\left\{\nu, m: 2^{-\nu}|m| \leq \varepsilon\right\}$, $\varepsilon$ small, only the powers $\alpha_{i}$ are relevant. By (4.3) we have

$$
2^{-\nu\left(s_{0}-s_{1}\right)} w_{0}\left(Q_{\nu, 0}\right)^{-\frac{1}{p_{0}}} w_{1}\left(Q_{\nu, 0}\right)^{\frac{1}{p_{1}}} \sim 2^{-\nu\left[s_{0}-\frac{d+\alpha_{0}}{p_{0}}-\left(s_{1}-\frac{d+\alpha_{1}}{p_{1}}\right)\right]},
$$

and for $m \neq 0$,

$$
2^{-\nu\left(s_{0}-s_{1}\right)} w_{0}\left(Q_{\nu, m}\right)^{-\frac{1}{p_{0}}} w_{1}\left(Q_{\nu, m}\right)^{\frac{1}{p_{1}}} \sim\left(2^{-\nu}|m|\right)^{\left[s_{0}-\frac{d+\alpha_{0}}{p_{0}}-\left(s_{1}-\frac{d+\alpha_{1}}{p_{1}}\right)\right]}|m|^{-\left[s_{0}-\frac{d}{p_{0}}-\left(s_{1}-\frac{d}{p_{1}}\right)\right]} .
$$

These expressions are bounded on $I_{1}$ if and only if the first two conditions in (4.2) are satisfied. Next consider indices $I_{2}=\left\{\nu, m: \varepsilon \leq 2^{-\nu}|m| \leq \varepsilon^{-1}\right\}$. Then

$$
w_{0}\left(Q_{\nu, m}\right) \sim w_{1}\left(Q_{\nu, m}\right) \sim|Q|=2^{-\nu d} .
$$

The boundedness of the expression in (C) on $I_{2}$ is thus equivalent to $s_{0}-\frac{d}{p_{0}} \geq s_{1}-\frac{d}{p_{1}}$. Finally, consider $I_{3}=\left\{\nu, m: 2^{-\nu}|m| \geq \varepsilon^{-1}\right\}$, where the powers $\beta_{i}$ are relevant. Using (4.3) for $m \neq 0$, we have

$$
2^{-\nu\left(s_{0}-s_{1}\right)} w_{0}\left(Q_{\nu, m}\right)^{-\frac{1}{p_{0}}} w_{1}\left(Q_{\nu, m}\right)^{\frac{1}{p_{1}}} \sim 2^{-\nu\left[s_{0}-\frac{d}{p_{0}}-\left(s_{1}-\frac{d}{p_{1}}\right)\right]}\left(2^{-\nu}|m|\right)^{\frac{\beta_{1}}{p_{1}}-\frac{\beta_{0}}{p_{0}}} .
$$

Here the boundedness on $I_{3}$ is equivalent to the second and third condition in (4.2). This proves the proposition.

4.2. Power weights acting radially in the first coordinates. A slight generalization of the above is the following. Let $d=n+k$ with $n, k \in \mathbb{N}$. Define for $\alpha, \beta>-n$ the weight $v_{\alpha, \beta}$ on $\mathbb{R}^{d}$ by

$$
v_{\alpha, \beta}(x, y)=w_{\alpha, \beta}(x), \quad x \in \mathbb{R}^{n}, \quad y \in \mathbb{R}^{k},
$$

where $w_{\alpha, \beta}$ is as in (4.1). Then $v_{\alpha, \beta}$ is a radial power weight in the first $n$ coordinates of $\mathbb{R}^{d}$ only and does not act on the last $k$ coordinates. An important example is the case $n=1$, where $v_{\alpha, \beta}$ is defined by powers of the distance to the hyperplane $\left\{x_{1}=0\right\}$.

One can check that the weighted cube volumes are given by

$$
v_{\gamma, \gamma}\left(Q_{\nu, m}\right) \sim 2^{-\nu(d+\gamma)}, \quad \pi_{n} m=0, \quad v_{\gamma, \gamma}\left(Q_{\nu, m}\right) \sim 2^{-\nu(d+\gamma)}\left|\pi_{n} m\right|^{\gamma}, \quad \pi_{n} m \neq 0,
$$

where $\pi_{n}$ projects onto the first $n$ coordinates. Distinguishing the sizes of $2^{-\nu}\left|\pi_{n} m\right|$, we thus obtain the same characterization of (C) as before.

Proposition 4.2. Let $d=n+k$ and $\alpha_{0}, \beta_{0}, \alpha_{1}, \beta_{1}>-n$. Then the weights

$$
v_{0}=v_{\alpha_{0}, \beta_{0}}, \quad v_{1}=v_{\alpha_{1}, \beta_{1}}
$$

satisfy (C) if and only if

$$
s_{0}-\frac{d+\alpha_{0}}{p_{0}} \geq s_{1}-\frac{d+\alpha_{1}}{p_{1}}, \quad s_{0}-\frac{d}{p_{0}} \geq s_{1}-\frac{d}{p_{1}}, \quad \frac{\beta_{0}}{p_{0}} \geq \frac{\beta_{1}}{p_{1}} .
$$

4.3. Products of power weights. Here we also refer to [4. Let $d=d_{1}+\ldots+d_{N}$ with $d_{j} \in \mathbb{N}_{0}$ for each $j=1, \ldots, N$. Let $w_{\alpha_{j}}$ be the weight on $\mathbb{R}^{d_{j}}$ defined by $w_{\alpha_{j}}(x)=|x|^{\alpha_{j}}$ for $\alpha_{j}>-d_{j}$. Denote by $\pi_{d_{j}}$ the projection of $\mathbb{R}^{d}$ onto the $d_{j}$ coordinates in $\mathbb{R}^{d}=\mathbb{R}^{d_{1}} \times \ldots \times \mathbb{R}^{d_{j}} \times \ldots \times \mathbb{R}^{d_{N}}$. Setting $\boldsymbol{\alpha}=\left(\alpha_{1}, \ldots, \alpha_{N}\right)$, define the weight $w_{\boldsymbol{\alpha}}$ on $\mathbb{R}^{d}$ by

$$
w_{\boldsymbol{\alpha}}(x)=\prod_{j=1}^{N} w_{\alpha_{j}}\left(\pi_{d_{j}} x\right) .
$$

Note that $w_{\boldsymbol{\alpha}} \in A_{p}$ if and only if $-d_{j}<\alpha_{j}<d_{j}\left(p_{j}-1\right)$ for $j=1, \ldots, N$. Arguing as before, for these types of weights one has the following.

Proposition 4.3. Let $d=d_{1}+\ldots+d_{N}$ with $d_{j} \in \mathbb{N}_{0}$ and consider as above $\boldsymbol{\alpha}=\left(\alpha_{1}, \ldots, \alpha_{N}\right)$ and $\tilde{\boldsymbol{\alpha}}=\left(\tilde{\alpha}_{1}, \ldots, \tilde{\alpha}_{N}\right)$ with $\alpha_{j}, \tilde{\alpha}_{j}>-d_{j}$ for $j=1, \ldots, N$. Then the weights $w_{0}=w_{\boldsymbol{\alpha}}$ and $w_{1}=w_{\tilde{\boldsymbol{\alpha}}}$ satisfy (C) if and only if

$$
s_{0}-\frac{d+\alpha_{1}+\ldots+\alpha_{N}}{p_{0}} \geq s_{1}-\frac{d+\tilde{\alpha}_{1}+\ldots+\tilde{\alpha}_{N}}{p_{1}}, \quad \frac{\alpha_{j}}{p_{0}} \geq \frac{\tilde{\alpha}_{j}}{p_{1}} \quad \text { for all } j=1, \ldots, N .
$$


Note that, since the weight exponents at zero and infinity are assumed to be the same, the condition $s_{0}-\frac{d}{p_{0}} \geq s_{1}-\frac{d}{p_{1}}$ is automatically satisfied.

4.4. Powers of the distance to a Lipschitz submanifold. Let $\Gamma$ be a $(d-k)$-dimensional compact Lipschitzian submanifold of $\mathbb{R}^{d}, 1 \leq k \leq d-1$. For $\gamma>-k$ let $w_{\gamma}(x)=\operatorname{dist}(x, \Gamma)^{\gamma}$. Then $w_{\gamma} \in A_{p}$ if and only if $-k<\alpha<k(p-1)$, see [8, Lemma 2.3]. Arguing by localization, we obtain the following characterization of (C), see also [7].

Proposition 4.4. Let $\gamma_{0}, \gamma_{1}>-k$. Then $w_{0}=w_{\gamma_{0}}$ and $w_{1}=w_{\gamma_{1}}$ satisfy (C) if and only if

$$
s_{0}-\frac{d+\gamma_{0}}{p_{0}} \geq s_{1}-\frac{d+\gamma_{1}}{p_{1}}, \quad \frac{\gamma_{0}}{p_{0}} \geq \frac{\gamma_{1}}{p_{1}} .
$$

4.5. The case $p_{1}<p_{0}$. For the above power-type weights, the condition (C) in particular yields sharp embeddings, in the sense that $s_{0}-s_{1}$ equals a certain optimal parameter depending on $w_{0}$, $w_{1}, p_{0}, p_{1}, q_{0}$ and $q_{1}$. In case $p_{1}<p_{0}$, for $F$ - and $H$-spaces such a sharp embedding does not hold, in general, as [18, Proposition 5.3] shows for radial power weights. For $w_{i}(x)=|x|^{\gamma_{i}}$ with $-d<\gamma_{i}<d\left(p_{i}-1\right), i=0,1$, we have $H^{s_{0}, p_{0}}\left(\mathbb{R}^{d}, w_{0}\right) \hookrightarrow H^{s_{1}, p_{1}}\left(\mathbb{R}^{d}, w_{1}\right)$ if and only if

$$
s_{0}-\frac{d+\gamma_{0}}{p_{0}}>s_{1}-\frac{d+\gamma_{1}}{p_{1}}, \quad \frac{d+\gamma_{0}}{p_{0}}>\frac{d+\gamma_{1}}{p_{1}} \text {. }
$$

\section{REFERENCES}

[1] H. Brezis and P. Mironescu. Gagliardo-Nirenberg, composition and products in fractional Sobolev spaces. J. Evol. Equ., 1(4):387-404, 2001.

[2] H.-Q. Bui. Weighted Besov and Triebel spaces: interpolation by the real method. Hiroshima Math. J., 12(3):581-605, 1982.

[3] H.-Q. Bui, M. Paluszyński, and M. H. Taibleson. A maximal function characterization of weighted BesovLipschitz and Triebel-Lizorkin spaces. Studia Math., 119(3):219-246, 1996.

[4] X. Cabré and X. Ros-Oton. Sobolev and isoperimetric inequalities with monomial weights. J. Differential Equations, 255(11):4312-4336, 2013.

[5] D.V. Cruz-Uribe, J. M. Martell, and C. Pérez. Weights, extrapolation and the theory of Rubio de Francia, volume 215 of Operator Theory: Advances and Applications. Birkhäuser/Springer Basel AG, Basel, 2011.

[6] P.L. De Nápoli, I. Drelichman, and N. Saintier. Weighted embedding theorems for radial Besov and TriebelLizorkin spaces. arXiv preprint arXiv:1406.0542, 2014.

[7] K. Disser, M. Meyries, and J. Rehberg. A unified framework for parabolic equations with mixed boundary conditions and diffusion on interfaces. arXiv preprint arXiv:1312.5882 2013.

[8] R. Farwig and H. Sohr. Weighted $L^{q}$-theory for the Stokes resolvent in exterior domains. J. Math. Soc. Japan, 49(2):251-288, 1997.

[9] J. Franke. On the spaces $\mathbf{F}_{p q}^{s}$ of Triebel-Lizorkin type: pointwise multipliers and spaces on domains. Math. Nachr., 125:29-68, 1986.

[10] J. García-Cuerva and J.L. Rubio de Francia. Weighted norm inequalities and related topics, volume 116 of North-Holland Mathematics Studies. North-Holland Publishing Co., Amsterdam, 1985. Notas de Matemática [Mathematical Notes], 104.

[11] L. Grafakos. Modern Fourier analysis, volume 250 of Graduate Texts in Mathematics. Springer, New York, second edition, 2009.

[12] D. D. Haroske and L. Skrzypczak. Entropy and approximation numbers of embeddings of function spaces with Muckenhoupt weights. I. Rev. Mat. Complut., 21(1):135-177, 2008.

[13] T.P. Hytönen. The two-weight inequality for the Hilbert transform with general measures. arXiv preprint arXiv:1312.0843, 2013.

[14] B. Jawerth. Some observations on Besov and Lizorkin-Triebel spaces. Math. Scand., 40(1):94-104, 1977.

[15] T. Kühn, H.-G. Leopold, W. Sickel, and L. Skrzypczak. Entropy numbers of embeddings of weighted Besov spaces. II. Proc. Edinb. Math. Soc. (2), 49(2):331-359, 2006.

[16] M.T. Lacey, E.T. Sawyer, I. Uriarte-Tuero, and C.-Y. Shen. Two weight inequality for the Hilbert transform: A real variable characterization, Part I. Duke Math. J., to appear, available at arXiv:1201.4319, 2012.

[17] M.T. Lacey and B.D. Wick. Two weight inequalities for Riesz transforms. arXiv preprint arXiv:1312.6163 2013.

[18] M. Meyries and M.C. Veraar. Sharp embedding results for spaces of smooth functions with power weights. Stud. Math., 208(3):257-293, 2012.

[19] M. Meyries and M.C. Veraar. Pointwise multiplication on vector-valued function spaces with power weights. Accepted by J. Fourier Anal. Appl., 2014.

[20] M. Meyries and M.C. Veraar. Traces and embeddings of anisotropic function spaces. Online first in Math. Ann., 2014. 
[21] F. Oru. Rôle des oscillations dans quelques problèmes d'analyse non-linéaire. PhD thesis, Doctorat de Ecole Normale Supérieure de Cachan.

[22] Y. Rakotondratsimba. A two-weight inequality for the Bessel potential operator. Comment. Math. Univ. Carolin., 38(3):497-511, 1997.

[23] V.S. Rychkov. Littlewood-Paley theory and function spaces with $A_{p}^{\text {loc }}$ weights. Math. Nachr., 224:145-180, 2001.

[24] E.T. Sawyer. A characterization of a two-weight norm inequality for maximal operators. Studia Math., 75(1):111, 1982.

[25] E.T. Sawyer, C.-Y. Shen, and I. Uriarte-Tuero. A geometric condition, necessity of energy, and two weight boundedness of fractional Riesz transforms. arXiv preprint arXiv:1310.4484, 2013.

[26] B. Scharf, H.-J. Schmeißer, and W. Sickel. Traces of vector-valued Sobolev spaces. Math. Nachr., 285(8-9):10821106, 2012.

[27] H.-J. Schmeisser and W. Sickel. Traces, Gagliardo-Nirenberg inequailties and Sobolev type embeddings for vector-valued function spaces. Jena manuscript, 2004.

[28] H.-J. Schmeißer and W. Sickel. Vector-valued Sobolev spaces and Gagliardo-Nirenberg inequalities. In Nonlinear elliptic and parabolic problems, volume 64 of Progr. Nonlinear Differential Equations Appl., pages 463-472. Birkhäuser, Basel, 2005.

[29] W. Sickel and H. Triebel. Hölder inequalities and sharp embeddings in function spaces of $B_{p q}^{s}$ and $F_{p q}^{s}$ type. Z. Anal. Anwendungen, 14(1):105-140, 1995.

[30] H. Triebel. Theory of function spaces, volume 78 of Monographs in Mathematics. Birkhäuser Verlag, Basel, 1983.

[31] M.C. Veraar. Embedding results for $\gamma$-spaces. Borichev, Alexander (ed.) et al., Recent trends in analysis. Proceedings of the conference in honor of Nikolai Nikolski on the occasion of his 70th birthday, Bordeaux, France, August 31 - September 2, 2011. Bucharest: The Theta Foundation. Theta Series in Advanced Mathematics, 209-219 (2013)., 2013.

M. Meyries, Institut für Mathematik, Martin-Luther-Universität Halle-Wittenberg, 06099 Halle (SaAle), Germany

E-mail address: martin.meyries@mathematik.uni-halle.de

M. Veraar, Delft Institute of Applied Mathematics, Delft University of Technology, P.O. Box 5031, 2600 GA Delft, The Netherlands

E-mail address: M.C.Veraar@tudelft.nl 